application of a sodium alginate solution is able to protect mucosal biopsies against impairment of oesophageal mucosal integrity when exposed to acidic solutions shortly after application. The durability of this protection is unclear.

We aimed to assess the protective effect and physical location of a topically applied sodium alginate solution $1 \mathrm{~h}$ after application.

Methods 3 mucosal biopsies were taken from the distal oesophagus $(3 \mathrm{~cm}$ above the $\mathrm{z}$-line) in 10 patients attending the Royal London Hospital for gastroscopy. Biopsies were transferred immediately to Krebs buffer $\mathrm{pH}$ 7.4. The luminal surfaces of 2 biopsies were coated with $200 \mu$ of either a sodium alginate solution (Gaviscon Advance, Reckitt Benckiser, Hull, UK) or a viscous control solution (of same viscosity, but without alginate). The biopsies were mechanically washed with $5 \mathrm{ml}$ Krebs, then each placed in an Using chambers and bathed in $\mathrm{pH} 7.4$ solution for $1 \mathrm{~h}$. The luminal aspect of the biopsy was then exposed for $30 \mathrm{~min}$ to an acidic solution $\mathrm{pH} 2+1 \mathrm{mg} / \mathrm{ml}$ pepsin $+1 \mathrm{mM}$ taurodeoxycholate. Percentage changes in TER from baseline at the end of exposure were recorded. For the $3^{\text {rd }}$ biopsy sodium alginate solution containing fluorescein-labelled alginate was used, and after $1 \mathrm{~h}$ bathing in $\mathrm{pH} 7.4$ solution the biopsy was fixed for immunohistological detection of the alginate.

Results Our previous experiments have demonstrated that exposure of unprotected biopsies to the acidic solution results in a $14.4 \pm 2.9 \%$ change in TER from baseline. $1 \mathrm{~h}$ after protection with alginate solution the same exposure caused a $-8.2 \pm 4.2 \%$ change in TER compared to $-15.9 \pm 3.0 \%$ change after protection with the viscous control $(\mathrm{p}<0.05)$.

Labelled alginate could be seen coating the luminal surface after $1 \mathrm{~h}$ in all cases.

Conclusion In vitro, alginate solutions can adhere to the oesophageal mucosa for up to $1 \mathrm{~h}$ and exert a topical protectant effect against refluxate-like solutions. This suggests that durable topical protectants can be further explored and developed as first-line/add-on therapies for GORD.

Disclosure of Interest P. Woodland: None Declared, C. Lee: None Declared, P. Dettmar Employee of: Technostics Limited, S. Preston: None Declared, D. Sifrim Grant/research support from: Reckitt-Benckiser, Sandhill Scientific, Consultant for: Sandhill Scientific.

\section{PTU-170 MISSED UPPER GASTROINTESTINAL (UGI) CANCERS AT ENDOSCOPY: A DISTRICT GENERAL HOSPITAL EXPERIENCE}

R Shakespeare*, M Maida. Department of Gastroenterology, Prince Charles Hospital, Merthyr Tydfil, UK

\subsection{6/gutjnl-2014-307263.244}

Introduction Despite advances in the staging and treatment, the prognosis of upper gastrointestinal tract (UGIT) cancer in the UK remains poor, often presenting insidiously at a late stage. However, in contrast to our understanding of missed colorectal cancer rates following colonoscopy, relatively few studies have been published addressing the frequency of missed UGIT malignancies. Depending on the population studied this ranges from 6.7 to $25.8 \%$. The aim of this study was to identify how frequently oesophagogastroduodenoscopy (OGD) may have failed to detect cancer at Prince Charles Hospital, a District General Hospital in South Wales, with a stable population of 150000 , in the 36 months preceding a confirmed histological diagnosis.
Methods All patients between $1^{\text {st }}$ January 2010 and $31^{\text {st }}$ December 2012 who underwent an OGD and were subsequently diagnosed with cancer were identified using endoscopic records and the cancer service database. Patients who had undergone a prior endoscopy within 3 years of diagnosis were then identified and their records reviewed to analyse the previous endoscopic and histological findings.

Results 5454 endoscopies were performed during this time period, and a total of 134 patients $(2.4 \%)$ with UGI cancer were identified. 77 (57\%) were oesophageal, 49 (37\%) gastric and 8 (6\%) duodenal. The mean age was 69 years (range 24-91), with a higher proportion of males to females $(3: 1)$. Of these, 9 patients $(6.7 \%)$ had undergone at least one previous endoscopy in the 36 months leading up to a confirmed UGIT cancer diagnosis, with $44 \%$ of these being within the preceding 12 months. The mean interval was 15 months. The majority (55\%) of patients had only one prior endoscopy (range 1-5). 8 patients $(89 \%)$ were found to have pathology at a preceding endoscopy at the site of a subsequently detected cancer. 6 patients were felt to have insufficient biopsy sampling $(<4$ or none) and 3 had inadequate surveillance or follow up of identified pathology (of which two had both inadequate sampling and surveillance).

Conclusion These findings, whilst similar to those previously reported in the literature have highlighted the importance of careful and thorough examination of the UGI tract, in particular with regard to adequate tissue sampling and surveillance. Consideration should be given to dedicated lists for surveillance of Barrett's and the use of additional techniques such as narrow band imaging and chromoendoscopy in order to enhance diagnostic accuracy.

Disclosure of Interest None Declared.

\section{PTU-171 RECURRENCE AFTER SUCCESSFUL RADIOFREQUENCY ABLATION FOR BARRETT'S RELATED NEOPLASIA IS MORE LIKELY IN MALES: DATA FROM THE UNITED KINGDOM PATIENT REGISTRY}

${ }^{1,2} \mathrm{RJ}$ Haidry ${ }^{*},{ }^{1} \mathrm{M}$ Banks, ${ }^{1} \mathrm{~A}$ Gupta, ${ }^{2} \mathrm{M}$ Butt, ${ }^{3} \mathrm{G}$ Fullarton, ${ }^{4} \mathrm{H}$ Smart, ${ }^{3} \mathrm{~J}$ Morris, ${ }^{5} \mathrm{R}$ Willert, ${ }^{6} \mathrm{R}$ Narayanasamy, ${ }^{1} \mathrm{M}$ Rodriguez-Justo, ${ }^{1} \mathrm{M}$ Novelli, ${ }^{1,2} \mathrm{~L}$ Lovat. ${ }^{1} \mathrm{UCLH}$, London, UK; ${ }^{2} N M L C$, UCL, London, UK; ${ }^{3} G R I$, Glasgow, UK; ${ }^{4} R L U H$, Liverpool, UK; ${ }^{5} M R I$, Manchester, UK; ${ }^{6}$ St James Hospital, Dublin, Ireland

\subsection{6/gutjnl-2014-307263.245}

Introduction Barrett's oesophagus (BE) can lead to oesophageal adenocarcinoma (OAC). BE is more prevalent in males. Endoscopic mucosal resection (EMR) for visible lesions followed by Radiofrequency ablation (RFA) have become first line treatment for patients with BE related neoplasia. Recurrence after treatment can occur in up to $25 \%$ of patients. Risk factors for recurrence are unclear.

Methods We examine prospective data from United Kingdom (UK) registry of patients undergoing RFA/EMR over past 5 years. We examine if recurrence after treatment is influenced by gender, baseline histology, BE length and prior EMR. Before RFA, visible lesions were removed by EMR. Thereafter patients underwent RFA 3 monthly. Biopsies were taken at 12 months and outcomes for clearance of dysplasia (CR-D) and BE (CR-IM) were assessed. After successful treatment patients were followed up 3 monthly for the first year, 6 monthly for second year and annually thereafter. Biopsies were taken from $1 \mathrm{~cm}$ below neo z-line and previously treated BE segment.

Results A total of 412 males and 95 females have been treated with no statistical difference in baseline BE length, histology or 